\title{
A Palmprint Verification System Based on Phase Congruency Features
}

\author{
Vitomir Štruc and Nikola Pavešić \\ Faculty of Electrical Engineering, \\ University of Ljubljana, \\ Tržaška 25, SI-1000 Ljubljana, Slovenia \\ \{vitomir.struc, nikola.pavesic\}@fe.uni-lj.si.com \\ http://luks.fe.uni-lj.si/
}

\begin{abstract}
The paper presents a fully automatic palmprint verification system which uses $2 \mathrm{D}$ phase congruency to extract line features from a palmprint image and subsequently performs linear discriminant analysis on the computed line features to represent them in a more compact manner. The system was trained and tested on a database of 200 people (2000 hand images) and achieved a false acceptance rate (FAR) of $0.26 \%$ and a false rejection rate (FRR) of $1.39 \%$ in the best performing verification experiment. In a comparison, where in addition to the proposed system, three popular palmprint recognition techniques were tested for their verification accuracy, the proposed system performed the best.
\end{abstract}

Key words: Palmprint verification, 2D phase congruency, Linear discriminant analysis

\section{Introduction}

Biometrics is a scientific discipline that involves methods of automatically recognizing (verifying or identifying) people by their physical and/or behavioral characteristics. Many biometric systems have already been presented in the literature, among them, systems which exploit biometric traits such as fingerprints, face, voice, iris, retina, hand-geometry, signature or palmprints are the most common [1].

Each of the listed biometric characteristics has its own strengths and weaknesses and is consequently more or less suited for a particular application domain. Face- and voice-based recognition systems, for example, are considered to be unintrusive, they do, however, still have problems achieving high recognition accuracy, especially when biometric samples (i.e., face images or speaker recordings) are captured in uncontrolled environments. Iris and retinal recognition, on the other hand, exhibit high recognition accuracy, but require intrusive acquisition systems [2]. Opposed to these recognition systems, palmprint-based recognition is considered both user-friendly as well as fairly accurate and thus provides an attractive alternative to other biometric systems. 
Existing (unimodal) palmprint recognition systems can according to [3] (based on the employed feature extraction technique) be classified into one of three groups: texture-based (e.g., [4]), line-based (e.g., [5,6]) and appearance-based (e.g., $[7,8])$. Though all feature types are relevant for palmprint-based biometric recognition, this paper focuses on line-based features.

Most of the palmprint recognition systems that make use of line features to verify the identity of a user employ gradient-based methods to extract characteristic lines from a palmprint image (e.g., [7, 8]). While these methods work fine on images of an appropriate quality (e.g., acquired in controlled illumination condition, free of distortions caused by the pressure applied to the surface of the scanner, etc.), they have problems when features have to be extracted from palmprint images of a poorer quality. In these situations a more robust approach is preferable. To this end, we have developed a palmprint verification system that uses line features extracted with the phase congruency model and is therefore relatively insensitive to image distortions caused by the acquisition procedure (note that images acquired with a desktop scanner almost always contain regions distorted by pressure - see Fig. 1).

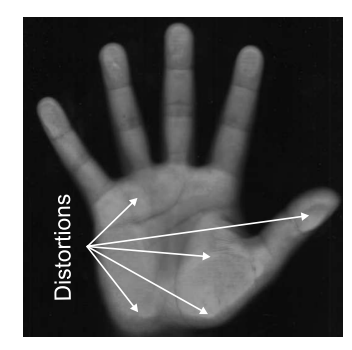

Fig. 1. Distortions of a palmprint image acquired with a desktop scanner

The rest of the paper is organized as follows: Section 2 gives a short description of the proposed palmprint verification system; Section 3 describes a series of verification experiments and presents their results; Section 4 concludes the paper with some final remarks and directions for future work.

\section{System Description}

The block diagram of the proposed palmprint recognition system is shown in Fig. 2. It is comprised of the following five modules: an acquisition module which uses a desktop scanner to capture an image of the palmar surface of the hand; a preprocessing module that extracts the region of interest (ROI), i.e., the palmprint region, from the acquired image and normalizes the extracted ROI in respect to size, rotation and illumination; a feature-extraction module which computes a set of phase congruency (PC) features from the normalized palmprint image and subsequently performs the linear discriminant analysis (LDA) on the feature 
set to enhance it's discriminatory power; a matching module that compares the computed feature set with a template (i.e., the mathematical representation of the feature sets extracted during the enrollment session) and outputs a matching score; and a decision module that uses the matching score to decide whether the person presented to the system is who he/she claims to be. A detailed description of each of the listed modules is given in the remainder of this section.

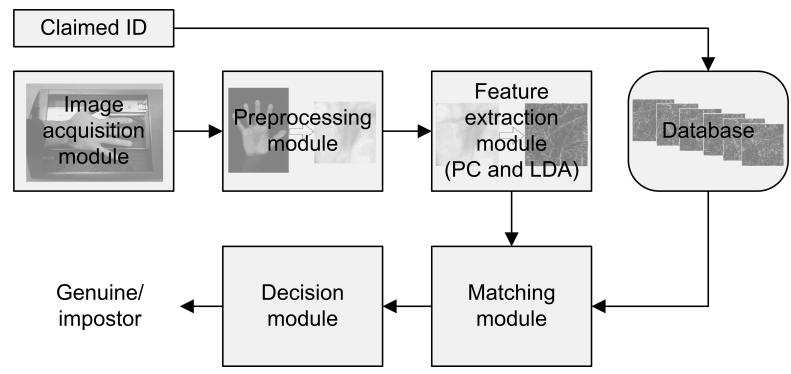

Fig. 2. The block diagram of the proposed palmprint recognition system

\subsection{Image acquisition}

The image-acquisition module of the proposed palmprint recognition system records grey-scale images of the palmar surface of the hand with the help of an optical desktop scanner rated at a resolution of 180 dots per inch (256 grey levels). When a person is presented to the system, he/she simply positions his/her hand on the scanner with the fingers spread naturally [1]. The system then acquires an image of the hand and passes it on to the preprocessing module.

\subsection{Image preprocessing}

After the acquisition stage, the acquired hand image is subjected to the preprocessing procedure which employs the following steps to extract and normalize the palmprint ROI from the hand image:

- Binarization: In the first step the hand region is extracted from the acquired grey-scale hand image (Fig. 3a) using an image thresholding procedure. Since a desktop scanner is employed in the acquisition stage the background of the image always appears as a black area in the image and the same (global) threshold can be used for binarization of all hand images (Fig. 3b).

- Contour extraction: In the second step the contour of the hand is extracted from the binarized hand image and used as the foundation for the palmprint localization procedure (an example of the extracted contour is shown in Fig. $3)$. 


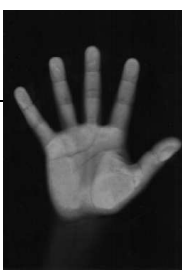

a)

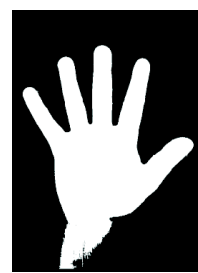

b)

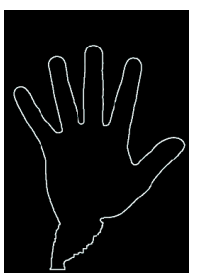

c)

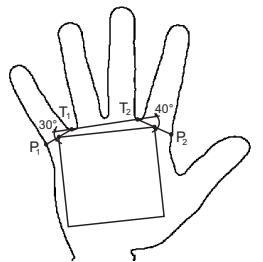

d)

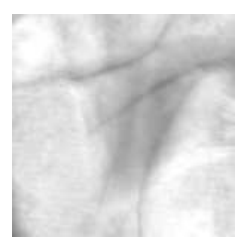

e)

Fig. 3. The preprocessing procedure: a) The hand image acquired by the desktop scanner, b) The binary image of the hand region, c) The image of the contour of the hand region, d) Extraction of the palmprint ROI, e) The normalized palmprint image

- ROI localization: To locate the palmprint ROI in the hand image, two reference points are determined in the third step of the preprocessing procedure. The first, denoted as $T_{1}$ in Fig. 3d, is located at the local minimum of the hand contour between the little and the ring finger, while the second, denoted as $T_{2}$ in Fig. 3 d, is set at the local minimum of the contour between the index and the middle finger. Based on the line connecting the reference locations $T_{1}$ and $T_{2}$ two additional points, i.e., $P_{1}$ and $P_{2}$, are determined on the hand contour as shown in Fig 3d. Finally, the palmprint ROI is located as the square region whose upper two corners correspond to the middle points of the line segments $P_{1}-T_{1}$ and $T_{2}-P_{2}[1,9]$.

- Normalization: In the last step the final palmprint ROI is obtained by rotating the cropped palmprint region to a predefined orientation and resizing it to a fixed size of $64 \times 64$ pixels. The geometrically normalized sub-image is ultimately subjected to an illumination normalization procedure which removes the mean of the pixel values from the grey-scale sub-image and subsequently scales all pixels with their standard deviation. An example of the normalized palmprint region is shown in Fig. 3e.

\subsection{Feature extraction}

The feature vector used in the matching procedure of the proposed system is extracted from the normalized palmprint image in two consecutive steps: in the first step, a set of 512 phase congruency features is computed from the input image and in the second step LDA is applied on this feature set to represent the phase congruency features in a discriminative and compact manner.

Phase congruency features. There have been a number of palmprint recognition systems presented in the literature that make use of line-based features, e.g., $[5,6]$. Typically, these systems use line detectors which scan the palmprint image for points of high intensity gradients to extract the line features. However, varying illumination conditions during the image acquisition stage (when images are captured with a camera-based sensor) or deformations of the palmprint region caused by pressure applied to the surface of the scanner (when images are 
captured with an optical scanner) often result in the detection of spurious lines. To avoid the listed difficulties, our systems employs the phase congruency model for line feature extraction.

The model searches for points in the palmprint sub-image where the $2 \mathrm{D}$ logGabor filter responses (of the sub-image) over several scales and orientations are maximally in phase $[10,11]$.

Let $\mathcal{G}=\left\{G\left(f_{h}, \theta_{g}\right): h=1,2, \ldots, p ; g=1,2, \ldots, r\right\}$ denote the set of 2D logGabor filters with $p$ scales and $r$ orientations and let $G\left(f_{h}, \theta_{g}\right)=G_{h g}$ be defined as:

$$
G_{h g}=\exp \left\{\frac{-\left[\ln \left(f / f_{h}\right)\right]^{2}}{2\left[\ln \left(k / f_{h}\right)\right]^{2}}\right\} \exp \left\{\frac{-\left(\theta-\theta_{g}\right)^{2}}{2 \sigma_{\theta}^{2}}\right\}
$$

where $f$ and $\theta$ denote the polar coordinates of the log-Gabor filter in the frequency domain, $f_{h}$ denotes the filters center frequency (in our experiments it was set to $\left.f_{h}=0.33 \cdot(2.1)^{1-h}\right), k$ defines the bandwidth of the filter in the radial direction (the ratio $k / f_{h}$ is commonly set to a constant value, for example, 0.55 like it was done in our case), $\theta_{g}=(g-1) \cdot \pi / r$ represents the orientation of the filter and $\sigma_{\theta}$ controls the angular bandwidth of the $2 \mathrm{D}$ log-Gabor filter (we used a value of $\left.\sigma_{\theta}=1.2 \cdot(\pi / r)\right)$.

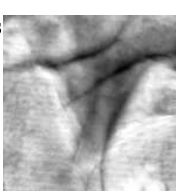

a)

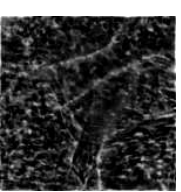

b)

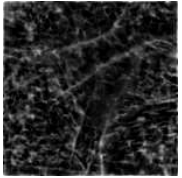

c)

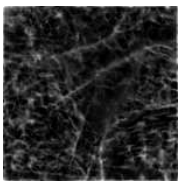

d)

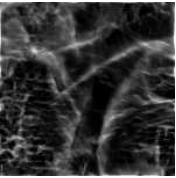

e)

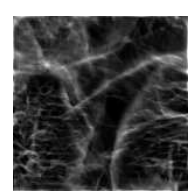

f)

Fig. 4. a) The normalized palmprint image; Phase congruency image for b) $p=3$ and $r=4$, c) $p=3$ and $r=6$, d) $p=3$ and $r=8$, e) $p=5$ and $r=6$, f) $p=5$ and $r=8$

Furthermore, let $I(x)$, where $x$ stands for the pixel location in the spatial domain, denote the grey-scale distribution of the normalized palmprint image (e.g., Fig. 3e). The magnitude $A_{h g}(x)$ and phase $\phi_{h g}(x)$ responses of the image $I(x)$ at a specific scale $h$ and orientation $g$ of the log-Gabor filter can then be computed as:

$$
\begin{aligned}
& A_{h g}(x)=\sqrt{\operatorname{Re}^{2}\left[I(x) * G_{h g}^{s}\right]+\operatorname{Im}^{2}\left[I(x, y) * G_{h g}^{s}\right]}, \\
& \phi_{h g}(x)=\arctan \left(\operatorname{Im}\left[I(x) * G_{h g}^{s}\right] / \operatorname{Re}\left[I(x) * G_{h g}^{s}\right]\right),
\end{aligned}
$$

where * denotes the convolution operator, $G_{h g}^{s}$ stands for the log-Gabor filter in the spatial domain at the scale $h$ and the orientation $g$ and $\operatorname{Re}[X]$ and $\operatorname{Im}[X]$ represent the real and imaginary parts of the convolution output.

Finally, the two-dimensional phase congruency features can according to [10] be computed using the following expression:

$$
P C_{2 D}(x)=\frac{\sum_{g} \sum_{h} W_{g}(x)\left\lfloor A_{h g}(x) \Delta \Phi_{h g}(x)-T_{g}\right\rfloor}{\sum_{g} \sum_{h} A_{h g}(x)+\varepsilon},
$$


where $T_{g}$ represents the estimated noise energy at orientation $g, W_{g}(x)$ denotes a weighting function that weights for the frequency spread, $\varepsilon$ is a small constant which prevents divisions by zero, the symbols $L\rfloor$ denote the following operation:

$$
\lfloor X-T\rfloor= \begin{cases}X-T & , \text { if } X>T \\ 0 & , \text { otherwise }\end{cases}
$$

and $\Delta \Phi_{h g}(x)$ is a phase deviation measure defined as:

$$
\Delta \Phi_{h g}(x)=\cos \left(\phi_{h g}(x)-\bar{\phi}_{g}(x)\right)-\left|\sin \left(\phi_{h g}(x)-\bar{\phi}_{g}(x)\right)\right| .
$$

In equation (6) $\phi_{h g}(x)$ denotes the phase angle at the location $x$ of the logGabor filter phase response at scale $h$ and orientation $g$, while $\bar{\phi}_{g}(x)$ represents the mean phase angle at the orientation $g$.

As we can see from the above discussion, phase congruency features are computed over multiple scales and orientation (using all filters from $\mathcal{G}$ ) making the feature extraction procedure robust to noise, illumination variations and image contrast. In addition to its robustness, the presented model also successfully explains the human perception of line (or edge) features [10].

Once a hand image is acquired, the palmprint sub-image extracted, properly normalized and transformed using the described phase congruency model, the final feature vector $\mathbf{x}$ is constructed by dividing the phase congruency image into a number non-overlapping blocks of size $4 \times 4$ pixels and then computing the mean value and standard deviation of the pixels in each of the 256 blocks (recall that we used palmprint images of size $64 \times 64$ pixels), i.e.,

$$
\mathbf{x}=\left(\mu_{1}, \sigma_{1}, \mu_{2}, \sigma_{2}, \ldots, \mu_{256}, \sigma_{256}\right)^{T} .
$$

However, as we can see from Fig. 4, the line features extracted with the phase congruency model vary in their appearance when log-Gabor filters with different numbers of scales and orientations are used. The effects of these parameters on the verification performance of the proposed system will be evaluated in Section 3.2 .

Linear discriminant analysis. Let us consider a set of $n d$-dimensional training phase congruency feature vectors $\mathbf{x}_{i}$ arranged in a $d \times n$ column data matrix $\mathbf{X}$, i.e., $\mathbf{X}=\left[\mathbf{x}_{1}, \mathbf{x}_{2}, \ldots, \mathbf{x}_{n}\right]$ and let us assume that each of the feature vectors belongs to one of $C$ classes (i.e., subjects - clients of the system). Based on the training data contained in the matrix $\mathbf{X}$, LDA first identifies a subspace (i.e., a subspace projection matrix $\mathbf{W}$ ) by maximizing a class separability criterion in the form of the ratio of the between-class to the within-class scatter matrix and then projects the phase congruency feature vectors into this subspace. The class separability criterion (sometimes called Fisher's discriminant criterion) is defined as follows [7]:

$$
J(\mathbf{W})=\frac{\left|\mathbf{W}^{T} \mathbf{S}_{B} \mathbf{W}\right|}{\left|\mathbf{W}^{T} \mathbf{S}_{W} \mathbf{W}\right|},
$$


where $\mathbf{S}_{B}$ and $\mathbf{S}_{W}$ denote the between-class and within-class scatter matrices defined as:

$$
\begin{gathered}
\mathbf{S}_{B}=\sum_{i=1}^{C} n_{i}\left(\boldsymbol{\mu}_{i}-\boldsymbol{\mu}\right)\left(\boldsymbol{\mu}_{i}-\boldsymbol{\mu}\right)^{T}, \\
\mathbf{S}_{W}=\sum_{i=1}^{C} \sum_{\mathbf{x}_{j} \in C_{i}}\left(\mathbf{x}_{j}-\boldsymbol{\mu}_{i}\right)\left(\mathbf{x}_{j}-\boldsymbol{\mu}_{i}\right)^{T},
\end{gathered}
$$

and the symbols $\boldsymbol{\mu}, \boldsymbol{\mu}_{i}, n_{i}$ and $C_{i}$ represent the global mean of all training feature vectors, the mean vector of the training feature vectors from the $i$-th class, the number of feature vectors in the $i$-th class and the label of the $i$-th class respectively.

It can be shown that the LDA transformation matrix $\mathbf{W}$ consists of the eigenvectors corresponding to the first $m \leq C-1$ largest eigenvalues of the following eigenproblem:

$$
\mathbf{S}_{W}^{-1} \mathbf{S}_{B} \mathbf{w}_{i}=\lambda_{i} \mathbf{w}_{i}, \quad i=1,2, \ldots, m
$$

Using the calculated transformation matrix $\mathbf{W}=\left[\mathbf{w}_{1}, \mathbf{w}_{2}, \ldots, \mathbf{w}_{m}\right]$ an arbitrary phase congruency feature vector $\mathbf{x}$ can be projected into the LDA subspace with the help of the following expression:

$$
\mathbf{y}=\mathbf{W}^{T} \mathbf{x} .
$$

However, in the field of palmprint recognition the number of training samples (i.e., training phase congruency feature vectors) per class is usually significantly smaller than the number of elements contained in each of the samples. This fact makes the matrix $\mathbf{S}_{W}$ singular (its' rank is at most $n-C$ ) and the computation of the transformation matrix $\mathbf{W}$ using equation (11) impossible. To overcome this problem, we first projected the matrices $\mathbf{S}_{B}$ and $\mathbf{S}_{W}$ into the principal component subspace to ensure that the matrix $\mathbf{S}_{W}$ is nonsingular and then performed LDA in this subspace. A detailed description of the employed approach can be found in $[7]$.

\subsection{Matching and decision}

At the matching stage the live feature vector $\mathbf{y}$ of a given input palmprint image computed with the help of the procedure described in the previous section is compared to the template $\overline{\mathbf{y}}_{i}$ associated with the claimed identity. The following similarity measure is used to produce the matching score:

$$
d\left(\mathbf{y}, \overline{\mathbf{y}}_{i}\right)=\frac{\left|\mathbf{y} \overline{\mathbf{y}}_{i}^{T}\right|}{\sqrt{\mathbf{y} \mathbf{y}^{T} \overline{\mathbf{y}}_{i} \overline{\mathbf{y}}_{i}^{T}}} .
$$

If the value of the normalized correlation coefficient defined by (13) is higher than the decision threshold the live feature vector and consequently the input palmprint image are recognized as genuine, otherwise they are recognized as belonging to an impostor. 


\section{Experiments}

\subsection{Database and experimental setup}

The proposed palmprint verification system was tested on hand-images of 200 subjects. During the acquisition stage each of the subjects was asked to position his/her hand on the desktop scanner 10 consecutive times, resulting in a database of 2000 images.

For testing purposes the subjects were randomly split into three groups, namely, the client group (120 subjects), the evaluation impostor group (30 subjects) and the test impostor group (50 subjects). Images belonging to subjects from the client group were further divided into sets of training images (4 per subject), evaluation images ( 3 per subject) and test images (3 per subject). Images from the client training set were used to construct client-templates (i.e., mean feature vectors), images from the impostor as well as the client evaluation set were used to compute the decision threshold and to optimize the system parameters (i.e., number of scales and orientations of the 2D log-Gabor filters) while the remaining test sets were employed exclusively for the final performance evaluation. During this last stage each of the 3 client test images was compared to the corresponding class in the database (a total of $3 \times 120=360$ experiments), whereas all 10 impostor test images were compared to each of the classes in the database (a total of $10 \times 50 \times 120=60,000$ experiments).

Three error rates were used in our experiments to rate the accuracy of the proposed palmprint verification system: the false acceptance rate (FAR) which measures the frequency of falsely accepted impostors, the false rejection rate (FRR) which measures the frequency of falsely rejected clients and the equal error rate (ERR) that is defined as the error rate at which the FAR and FRR are equal. In addition to providing an accuracy measure for the proposed system, the ERR (obtained on the evaluation sets) was used for determining the decision threshold.

\subsection{Parameter tuning}

Our first set of experiments assessed the performance of the proposed palmprint verification system with respect to the number of scales and orientations of the 2D log-Gabor filters used to compute the phase congruency features. The system was tested for 5 different combinations of the values of $p$ and $r$ (see Section 2.3). In all cases the number of features was set to its maximal value, i.e., $m=119$. The results of the experiments are presented in Fig. 5 and Table 1 which show the ROC curves and the values of the FAR and FRR at the ERR operating point respectively.

As we can see, varying the number of filter orientations had only a small effect on the verification performance of the proposed system. Larger differences were detected when the number of scales was changed. Furthermore we can notice that the error rates at the equal error operating point for images processed with $\log$-Gabor filters at 3 scales and different numbers of orientations are virtually 


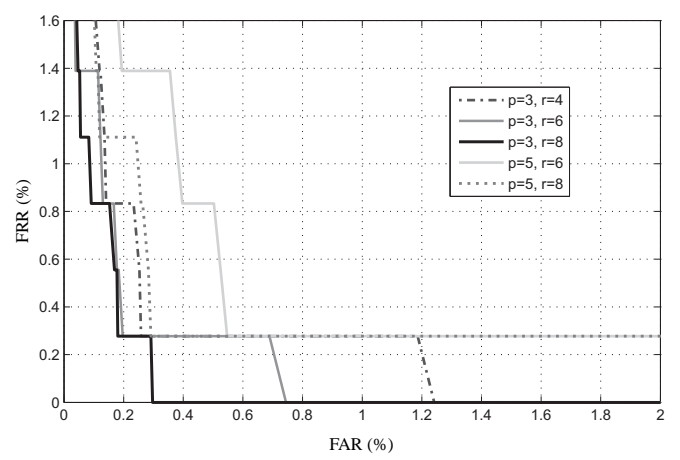

Fig. 5. The ROC curves of the performed experiments

Table 1. The FRRs and FARs of the experiments at the equal error operating point

\begin{tabular}{|c|c|c|c|}
\hline No. of scales & No. of orient. & $F A R(\%)$ & $F R R(\%)$ \\
\hline \hline$p=3$ & $r=4$ & 0.26 & 0.28 \\
\hline$p=3$ & $r=6$ & 0.26 & 0.28 \\
\hline$p=3$ & $r=8$ & $\mathbf{0 . 2 5}$ & $\mathbf{0 . 2 8}$ \\
\hline$p=5$ & $r=6$ & 0.50 & 0.83 \\
\hline$p=5$ & $r=8$ & 0.28 & 0.56 \\
\hline
\end{tabular}

the same. However, by looking at Fig 5 we can see that the combination of 3 scales and 8 orientations performed the best (considering all possible operating points).

\subsection{Performance evaluation}

The goal of the second set of verification experiments was to assess the performance of the proposed system on an independent set of test images. Additionaly, three popular palmprint-feature extraction techniques were implemented, trained and compared to our approach. Specifically the following methods were implemented for comparison: the eigenpalm approach [8], the fisherpalm apporach [7] and a line-feature [5] based approach (denoted as LFBA in Table 2) in combination with LDA. Note, however, that the original LFBA, i.e., as presented in [11], does not use LDA to extract the final palmprint features. LDA was added to allow for a fair comparison with the proposed approach which also includes a LDA step.

The results of the experiments in terms of the FRR and FAR obtained with the threshold that ensured equal error rates on the evaluation set are presented in Table 2. Two findings should be emphasized based on the these results: first, the FRRs of all methods increased in the final testing stage, most likely due to an unrepresentative training set which did not account for all possible variations in the appearance of the line features of the client images; and second, the proposed line features resulted in the best verification performance of all tested methods. 
Table 2. Comparison of the FRRs and FARs for different feature extraction techniques

\begin{tabular}{|c|c|c|}
\hline Feature extraction procedure & $F A R(\%)$ & $F R R(\%)$ \\
\hline \hline Eigenpalm & 2.94 & 3.61 \\
\hline Fisherpalm & 0.30 & 1.94 \\
\hline LFBA & 0.39 & 2.22 \\
\hline Proposed approach & $\mathbf{0 . 2 6}$ & $\mathbf{1 . 3 9}$ \\
\hline
\end{tabular}

\section{Conclusion and future work}

We have presented a palmprint recognition system that used phase congruency and linear discriminant analysis to extract discriminative palmprint features. The system was tested on a database of 2000 hand images and achieved a false acceptance rate of $0.26 \%$ and a false rejection rate of $1.39 \%$ using the decision threshold that ensured equal error rates on an independent evaluation set. Based on these encouraging results, our future work will be focused on the integration of phase congruency features into a multi-modal (i.e., intra-modal) palmprint recognition system.

\section{References}

1. Pavešić, N., Ribarić, S., Ribarić, D.: Personal Authentication Using Hand-Geometry and Palmprint Features - The State of the Art. In: Proceedings of the Workshop: Biometrics - Challenges Arising from Theory to Practice, pp. 17-26. Cambridge (2004)

2. Yoruk, E., Dutagaci, H., Sankur, B.: Hand Biometrics. Image and Vision Computing 24(5), 483-497 (2006)

3. Kumar, A., Zhang, D.: Personal Authentication Using Multiple Palmprint Representation. Pattern Recognition 38(10), 1695-1704 (2005)

4. Zhang, D., Kong, W.K., You, J., Wong, M.: Online Palmprint Identification. IEEE Transactions on Pattern Analysis and Machine Intelligence 25(9), 1041-1050 (2003)

5. Zhang, D., Shu, W.: wo Novel Characteristics in Palmprint Verification: Datum Point Invariance and Line Feature Matching. Pattern Recognition 32(4), 691-702 (1999)

6. Kumar, A., Wong, D.C.M., Shen, H.C., Jain, A.K.: Personal Authentication Using Hand Images. Pattern Recognition Letters 27(13), 1478-1486 (2006)

7. Wu, X., Zhang, D., Wang, K.: Fisherpalms Based Palmprint Recognition. Pattern Recognition Letters 24(15), 2829-2838 (2003)

8. Lu, G., Zhang, D., Wang, K.: Palmprint Recognition Using Eigenpalm Features. Pattern Recognition Letters 24(9-10), 1463-1467 (2003)

9. Ribarić, S., Fratrić, I.: A Biometric Identification System Based on Eigenpalm and Eigenfinger Features. IEEE Transactions on Pattern Analysis and Machine Intelligence 27(11), 1698-1709 (2005)

10. Kovesi, P.: Image Features From Phase Congruency. Videre: Journal of Computer Vision Research 1(3), 1-26 (1999)

11. Gundimada, S., Asari, V.K.: A Novel Neighborhood Defined Feature Selection on Phase Congruency Images for Recognition of Faces with Extreme Variations. International Journal of Information Technology 3(1), 25-31 (2006) 